\title{
A study on lateral speed estimation methods
}

\author{
Ali Y. Ungoren and Huei Peng* \\ Department of Mechanical Engineering, University of Michigan, \\ 2250 G.G. Brown, Ann Arbor, MI 48109-2125, USA \\ E-mail: hpeng@umich.edu \\ *Corresponding author

\section{H.E. Tseng}

Scientific Research Laboratory, Ford Motor Company

E-mail: htseng@ford.com

\begin{abstract}
The estimation of vehicle lateral speed, a critical variable for vehicle stability control, four-wheel-steering and other advanced dynamic control systems, is studied in this paper. We presented three different approaches, one each from three categories: transfer function approach, state-space approach, and kinematics approach. The first two methods rely on a vehicle dynamic (bicycle) model, and the last approach is based on the kinematics relationship of measured signals. The basic formulation of all three methods assumed that the road bank angle is negligible, and thus needs to be enhanced by a road bank angle estimation algorithm to work satisfactorily when the road bank is significant. The performance of these three (enhanced) methods are investigated using simulation and experimental data. For the experimental verification, we present four cases: nominal (high friction, flat road), banked road, low-friction, and low-friction-near-spin. Weakness of the three estimation algorithms is discussed.
\end{abstract}

Keywords: active safety; active yaw control; lateral velocity estimation; side slip angle estimation; vehicle stability control.

Reference to this paper should be made as follows: Ungoren, A.Y., Peng, H. and Tseng, H.E. (2004) 'A study on lateral speed estimation methods', Int. J. Vehicle Autonomous Systems, Vol. 2, Nos. 1/2, pp.126-144.

Biographical notes: Ali Yigit Ungoren received his B.S. degree in Mechanical Engineering from Bogazici University, Istanbul, Turkey, in 1997, and the M.S. and Ph.D. degrees in Mechanical Engineering from the University of Michigan, Ann Arbor, in 1999 and 2003, respectively. His research interest includes worst-case analysis, optimal control and vehicle dynamics. Currently he is working for Kores Metal A.S. as a technical coordinator.

Huei Peng is currently an Associate Professor of the Department of Mechanical Engineering, and the Director of the Automotive Engineering Program, at the University of Michigan, Ann Arbor. His research interests include adaptive control and optimal control, with emphasis on their applications to vehicular and transportation systems. He has been an active member of SAE, the ASME Dynamic System and Control Division. $\mathrm{He}$ served as chair of the ASME DSCD Transportation Panel from 1995 to 1997. He is currently an Associate Editor for the IEEE/ASME Transactions on Mechatronics. He received the National Science Foundation (NSF) Career award in 1998.

Copyright (C) 2004 Inderscience Enterprises Ltd. 
Hongtei Eric Tseng received his B.S. degree from National Taiwan University, Taipei, Taiwan, the M.S. and Ph.D. degrees from the University of California at Berkeley, in 1986, 1991 and 1994, respectively, all in Mechanical Engineering. In 1994, he joined Ford Motor Company. $\mathrm{He}$ is the holder of ten US patents. His previous work includes a low tyre pressure warning system using wheel speed sensors; vehicle yaw stability control and roll stability control using brake and powertrain actuations, and development of advanced vehicle dynamics observers. He is currently a Staff Technical Specialist at Powertrain Controls, Research and Development, Ford Research and Advanced Engineering. $\mathrm{He}$ is a member of the American Society of Mechanical Engineers (ASME).

\section{Introduction}

Lateral speed is one of the most important vehicle dynamic variables for Vehicle Stability Control (VSC) systems and is also crucial for other chassis control functions such as four-wheel-steering. While vehicle lateral speed can be directly measured by instrumental sensors such as optical sensors or GPS sensors, there are practical issues such as cost, accuracy and reliability that inhibit production vehicles from using these sensors at present or in the near future. Therefore, the estimation of vehicle lateral speed based on other vehicle input/output signals is an important topic and has been widely discussed in the literature.

Senger and Kortum [1] developed a vehicle handling model and tyre model to estimate the lateral speed. Their approach assumed that the tyres were operating in the linear region with known cornering stiffness. Cao [2] used a combined parameter and state estimation approach to accommodate unknown and varying cornering stiffness. However, the effectiveness of Cao's method with steering angle, vehicle speed and lateral acceleration as the only measurements is doubtful (from our experience) and the method is unlikely to overcome the challenges that arise from road bank variations. Kaminaga and Naito [3] applied a standard Lyapunov-based adaptive observer technique for side-slip estimation. The algorithm was found to provide satisfactory robustness for cornering stiffness variation on flat road surfaces. Experimental results provided in their paper show that the side slip angle estimation error is roughly below 3 degrees. Liu and Peng [4] proposed a different identification scheme for simultaneous state and parameter estimation to overcome the unknown vehicle parameters. However, the observer stability is only proven for the case of time invariant cornering stiffness and/or road surfaces. It is further observed in their paper that the proposed methodology requires one to two cycles of vehicle manoeuvres before the estimation converges to satisfactory performance with time invariant cornering stiffness. Its performance under real driving conditions has not been adequately verified.

Farrelly and Wellstead [5] proposed both a physical modelling approach as well as a kinematics modelling approach. In their physical modelling approach, the observer can be tuned to perform despite certain parameter changes. For example, it can be designed to be robust under cornering stiffness variation in the front axle, or in the rear axle. However, the observer will not work reliably when both front and 
rear cornering stiffness are unknown and/or varying. As for their kinematics approach, the observer has been shown to provide satisfactory performance whenever the vehicle yaw rate is non-zero. Fukada [6] suggested a combined observer/direct integration method. The method strove to balance the robustness of modelling error (provided by direct integration) and that of signal bias (provided by tyre model feedback). A road bank/slant estimate was also provided. In this dual approach, the lateral velocity estimate depends on the road bank estimate that, in turn, depends on the lateral velocity estimate. While this may 'result in a complex system and a risk of instability', the issues were empirically resolved. The experimental results on flat road show excellent performance in a double lane change manoeuvre but significant error (about $40 \%$ less than the actual) in a J-turn manoeuvre. The J-turn manoeuvre on snow is inherently difficult for this approach since the signal to noise ratio was small (as vehicle side drifted slowly) and the tyres were in non-linear regions. This method was subsequently refined, and integrated into a Vehicle Stability Control system [7]. The control results seem to suggest that the estimation was improved and is satisfactory for VSC.

Sasaki and Nishimaki [8] suggested a neural network method for side-slip angle estimation. The proposed form of the $\mathrm{I} / \mathrm{O}$ relationship is $\beta(k+1)=F(r(k), \ldots$, $r(k-n), a y(k), \ldots, a y(k-n))$, where $\beta, r, a y$ are vehicle side slide angle, yaw rate and lateral acceleration, respectively. This equation seems fundamentally flawed because vehicle longitudinal speed greatly influences vehicle lateral dynamics and thus the omission of this key variable severely limited the credible range of the above equation. Furthermore, when there is any change in tyre/road friction characteristics, or when the road is banked, this neural-network solution, which is feed-forward in nature, will not work satisfactorily.

Hac and Simpson [9] developed an algorithm that uses wheel speeds and vehicle lateral acceleration measurement, and a friction coefficient estimation scheme enhanced nonlinear vehicle model to estimate yaw rate and lateral speed simultaneously. The experimental results, especially those on icy surfaces, are impressive. However, the effect of another important factor - road bank angle, is not adequately addressed.

In summary, the lateral speed (or side slip angle) estimation problem has been actively studied over the last decade. However, there have been few comparison studies between representative methods, and thus it will be the main focus of this paper. We will focus on the discussion and comparison of three representative methodologies: a transfer function approach, the state-space approach by Liu and Peng [4], and the kinematics approach by Farrelly and Wellstead [5]. First, simulations are performed under ideal conditions to see their best case performance by using clean data generated by the TruckSim ${ }^{(i)}$ software (www.trucksim.com). It is understood that since the standard bicycle model does not include the effect of the road bank angle, any algorithm that is developed based on the original bicycle model may not be robust against road bank angle perturbation (which is the case for all three methods). Therefore, a road bank estimation 'plug-in' is introduced to enhance the performance of these three methods under a banked-road scenario.

Subsequently, performance and robustness of the three 'enhanced' algorithms under real-world driving situations are studied by post-processing experimental vehicle test data from various road and manoeuvre conditions. In particular, we 
focus on performance evaluation of these algorithms on low friction or banked road surfaces. The comparison of the three approaches, especially their weakness, will then be discussed.

\section{Estimation methods}

\subsection{Transfer function approach}

The basis of this estimation method is the bicycle vehicle model, which describes the vehicle lateral and yaw dynamics of a 2-axle, 1-rigid body ground vehicle (see Figure 1). The bicycle model can be represented in the form

$$
\left[\begin{array}{c}
\dot{v} \\
\dot{r}
\end{array}\right]=\left[\begin{array}{cc}
-\frac{1}{m}\left(\frac{2 C_{f}+2 C_{r}}{v_{x}}\right) & \frac{1}{m}\left(-m v_{x}+\frac{-2 a C_{f}+2 b C_{r}}{v_{x}}\right) \\
\frac{1}{I_{z}}\left(\frac{-2 a C_{f}+2 b C_{r}}{v_{x}}\right) & \frac{1}{I_{z}}\left(\frac{-2 a^{2} C_{f}-2 b^{2} C_{r}}{v_{x}}\right)
\end{array}\right]\left[\begin{array}{l}
v \\
r
\end{array}\right]+\left[\begin{array}{c}
\frac{2 C_{f}}{m} \\
\frac{2 a C_{f}}{I_{z}}
\end{array}\right] \delta_{f}
$$

where $v_{x}$ is the vehicle forward speed, $v$ is the vehicle lateral speed, $r$ is the yaw rate, $m$ is the vehicle mass, $I_{z}$ is the yaw moment of inertia, $C_{f}$ and $C_{r}$ are the front and rear cornering stiffness (per tyre). $\delta_{f}$ is the front wheel steering angle, and $a$ and $b$ are the distance from vehicle centre of gravity to front and rear axles, respectively.

Figure 12 DOF (bicycle) model

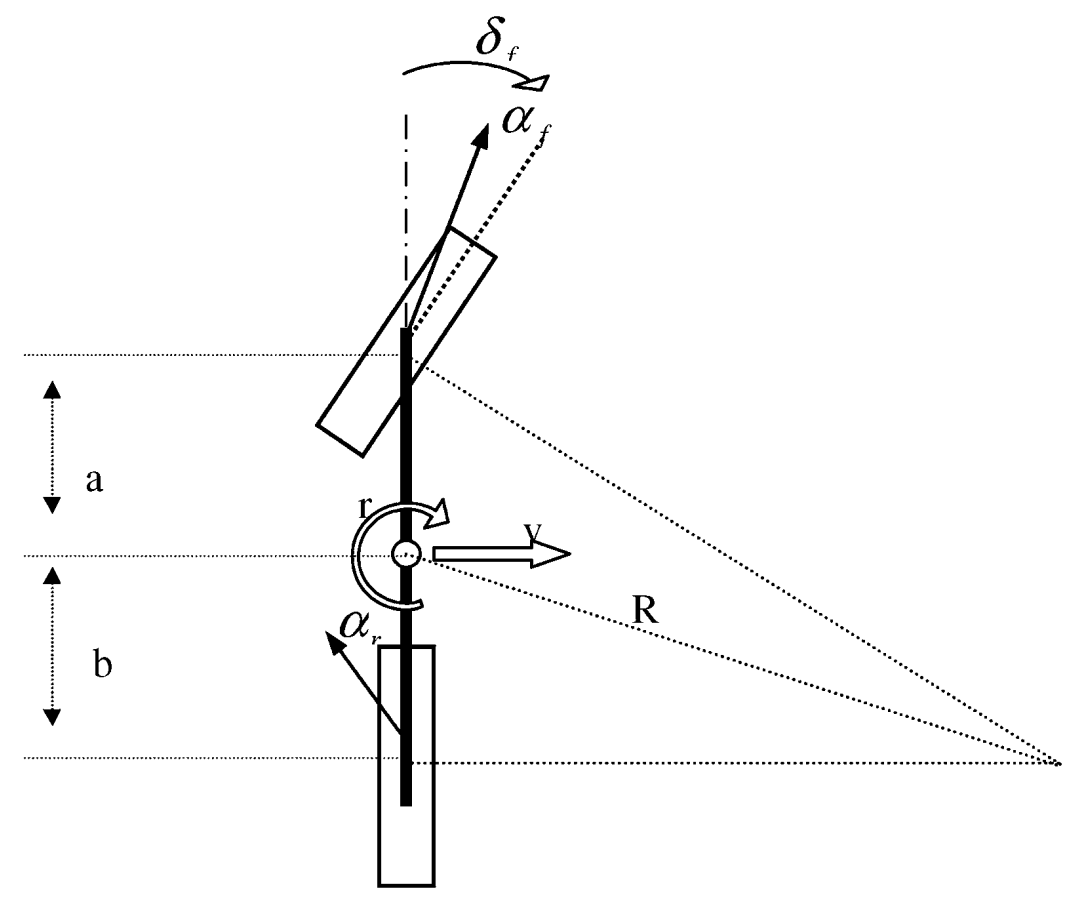


If we re-write Equation (1) in a simpler form:

$$
\left[\begin{array}{c}
\dot{v} \\
\dot{r}
\end{array}\right]=\left[\begin{array}{ll}
a_{1} & a_{3} \\
a_{2} & a_{4}
\end{array}\right]\left[\begin{array}{l}
v \\
r
\end{array}\right]+\left[\begin{array}{l}
b_{1} \\
b_{2}
\end{array}\right] \delta_{f}
$$

then it is straightforward to write the transfer functions from steering to the two states as

$$
H_{\delta \rightarrow v}=\frac{s b_{1}-a_{4} b_{1}+a_{3} b_{2}}{s^{2}-s\left(a_{1}+a_{4}\right)+a_{1} a_{4}-a_{2} a_{3}}
$$

and

$$
H_{\delta \rightarrow r}=\frac{s b_{2}+a_{2} b_{1}-a_{1} b_{2}}{s^{2}-s\left(a_{1}+a_{4}\right)+a_{1} a_{4}-a_{2} a_{3}} .
$$

The basic idea of the transfer function method is as follows: we can first use the available measurements (steering angle and yaw rate) to obtain a least-square fit for the transfer function shown in Equation (4) in real time. This optimal ARMA model, which best describes the vehicle yaw motion in the discrete-time format, will then be converted into the continuous-time where best-guess parameters of the transfer function for the yaw rate output (Equation (4)) can be obtained. Since the parameter $b_{2}$, which is the coefficient of the 's' term of the numerator polynomial of Equation (4), is related to the front tyre cornering stiffness in a simple form $\left(b_{2}=\frac{2 a C_{f}}{I_{z}}\right.$, see Equation (1)), it is straightforward to calculate a best-guess front cornering stiffness, $C_{f}$, the value of which can be used to compute $b_{1}=\frac{2 C_{f}}{m}$. These values can then be plugged into the constant term of the numerator polynomial of Equation (4), $a_{2} b_{1}-a_{1} b_{2}$, to calculate the rear tyre cornering stiffness $C_{r}$. Finally, using the computed tyre cornering stiffness values, the numerator polynomial of the lateral speed transfer function shown in Equation (3) can be calculated. Since the two transfer functions share the same denominator, the transfer function in Equation (3) is completely known, based on which the vehicle lateral speed can be estimated.

Since the discrete time ARMA identification process requires only measured variables in three history steps, the adaptation for tyre/road characteristics change is expected to be fast and thus a responsive estimation can be obtained. It is important to point out that the basic idea of this approach is simple. In practice, we frequently found that the identified least-square model becomes unstable or very lightly damped, especially when the vehicle yaw rate is rising quickly, responding to fast steering input. Under these circumstances, we will discard these unstable models, and just keep using the non-updated (but stable and well-damped) model as our best guess. This modification is necessary to keep the estimated tyre cornering stiffness positive, and to ensure that the model prediction is reasonable. This threshold value was found to have a significant effect on the final estimation results.

\subsection{State-space approach}

The idea presented in the previous section can be easily extended to construct a state-space method, based on which vehicle lateral speed can be estimated. However, this two-step approach relies heavily on the structure of the transfer function and 
might be very sensitive to uncertainties, such as variation in other vehicle parameters ( $m, I_{z}$, etc.) The adaptive algorithm developed by Liu and Peng [4] uses a one-step strategy. The algorithm was developed to estimate states and unknown parameters simultaneously for nonlinear time invariant systems which depend affinely on the unknown parameters. The lateral speed estimation problem was used in their paper as a case study example to show the possibility to estimate vehicle lateral speed under uncertain road friction conditions. Due to the fact that tyre cornering stiffness of the two axles is generally unknown and are the main source of model uncertainties, Equation (1) can be rewritten to reflect the need to estimate these unknown parameters. The linearly parameterised form of Equation (1) is

$$
\begin{aligned}
& \dot{x}=A x+u \theta \\
& y=r
\end{aligned}
$$

where

$$
x=\left[\begin{array}{l}
v \\
r
\end{array}\right], A \equiv\left[\begin{array}{ll}
a_{1} & a_{3} \\
a_{2} & a_{4}
\end{array}\right],
$$

$u$ is the front axle steering angle, and

$$
\theta=\left[\begin{array}{ll}
\frac{2 C_{f}}{m} & \frac{2 a C_{f}}{I_{z}}
\end{array}\right]^{T} .
$$

The adaptive observer is then

$$
\dot{\hat{x}}=\left(A_{n}+\Delta \hat{A}_{x}\right) \hat{x}+\Delta \hat{A}_{y} r+\left[\begin{array}{c}
-v_{x} \\
0
\end{array}\right] r+u \hat{\theta}+K(y-\hat{y})
$$

where

$$
\hat{x}=\left[\begin{array}{ll}
\hat{v} & \hat{r}
\end{array}\right]^{T}, \hat{y}=\hat{r}, A_{n}=\left[\begin{array}{cc}
-\left(\frac{2 C_{f n}+2 C_{r n}}{v_{x} m_{n}}\right) & \left(\frac{-2 a_{n} C_{f n}+2 b_{n} C_{r n}}{v_{x} m_{n}}\right) \\
\left(\frac{-2 a_{n} C_{f n}+2 b_{n} C_{r n}}{v_{x} I_{z n}}\right) & \left(\frac{-2 a_{n}^{2} C_{f n}-2 b_{n}^{2} C_{r n}}{v_{x} I_{z n}}\right)
\end{array}\right],
$$

$$
\Delta \hat{A}_{x}=\left[\begin{array}{ll}
\Delta \hat{a}_{1} & 0 \\
\Delta \hat{a}_{2} & 0
\end{array}\right] \text { and } \Delta \hat{A}_{y}=\left[\begin{array}{ll}
\Delta \hat{a}_{3} & \Delta \hat{a}_{4}
\end{array}\right]^{T} \text {. }
$$

All the variables with a subscript ' $n$ ' denote the nominal values of that variable. For example, $C_{f n}$ is the nominal value of $C_{f}$, and so forth. The unknown variables $\Delta \hat{a}_{i}$ denote the deviation of $a_{i}$ from their nominal values (entries in the $A_{n}$ matrix), which are to be estimated. If the observer gain is selected as $K=\frac{\left[k_{1}, k_{2}\right]^{T}}{v_{x}}$, i.e.

$$
A_{n}-K C=\frac{1}{v_{x}}\left[\begin{array}{cc}
-\left(\frac{2 C_{f n}+2 C_{r n}}{m_{n}}\right) & \left(\frac{-2 a_{n} C_{f n}+2 b_{n} C_{r n}}{m_{n}}\right)-k_{1} \\
\left(\frac{-2 a_{n} C_{f n}+2 b_{n} C_{r n}}{I_{z n}}\right) & \left(\frac{-2 a_{n}^{2} C_{f n}-2 b_{n}^{2} C_{r n}}{I_{z n}}\right)-k_{2}
\end{array}\right]
$$


where $C=\left[\begin{array}{ll}0 & 1\end{array}\right]$, then the closed-loop state matrix is stable when $k_{1}$ and $k_{2}$ are properly selected, according to the nominal vehicle parameters. When $\Delta A_{x}, \Delta A_{y}$ and $\theta$ are constant (slow varying), these unknown parameters, as well as the state variables are estimated by using a standard state observer (which uses estimated parameters for its model), together with a parameter update law, which is gain-varied based on the calculation of a set of augmented states. The equations are too numerous to recite completely in this paper. Interested readers are referred to the original paper by Liu and Peng for details [4]. This estimator, like most other adaptive algorithms, requires a persistent excitation condition to achieve small estimation errors. In other words, when the vehicle is driving straight, the vehicle parameters will not be updated accurately. In other words, if the transition from high friction to low friction road surfaces occur during a straight section of the roadway, this algorithm will need some time to correct its estimation, and thus it might suffer slow convergence transiently. The most important design parameters of this estimator were found to be the observer gains $k_{1}$ and $k_{2}$, which need to be tuned properly according to the trade-off between noise rejection and convergence speed. We also found it necessary to constrain the estimated parameter values, to ensure that they stay within reasonable values governed by the physical parameters.

\subsection{Kinematics approach}

The kinematics approach proposed by Farrelly and Wellstead [5] is very promising as the stability of the observer/convergence of estimation error has been shown for all non-zero yaw rates. In this paper we integrated their methodology with a physical model based observer to avoid unobservability and the drifting issues (as described by Farrelly and Wellstead) [5] during near-zero yaw rate conditions. The implemented observer arbitrates between the kinematics model based observer and a physical model based observer by using a yaw rate criterion. The kinematics model keeps track of $u$ and $v$, the vehicle longitudinal and lateral speeds according to their kinematics relationship with their derivatives. The model is described in the following:

$$
\text { If }\left(|r|>r_{c} \operatorname{deg} / \mathrm{sec}\right)
$$

$$
\left[\begin{array}{c}
\dot{\hat{v}}_{x} \\
\dot{\hat{v}}
\end{array}\right]=\left[\begin{array}{cc}
0 & r \\
-r & 0
\end{array}\right]\left[\begin{array}{c}
\hat{v}_{x} \\
\hat{v}
\end{array}\right]+\left[\begin{array}{l}
a_{x} \\
a_{y}
\end{array}\right]+\left[\begin{array}{l}
k_{1} \\
k_{2}
\end{array}\right]\left(\hat{v}_{x}-v_{x}\right)
$$

where

$$
k_{1}=2|\alpha| r, \quad k_{2}=\left(\alpha^{2}-1\right) r
$$

else

$$
\dot{\hat{v}}=-v_{x} r+\hat{a}_{y} \quad \hat{v}_{x}:=v_{x}
$$

where

$$
\hat{a}_{y}=\left[2 C_{f}\left(\tan ^{-1}\left(\frac{\hat{v}+a r}{v_{x}}\right)-\delta\right)+2 C_{r} \tan ^{-1}\left(\frac{\hat{v}-b r}{v_{x}}\right)\right] / m .
$$


Note that $r_{c}$ and $\alpha$ are design parameters and $\delta, v_{x}, r, a_{x}$, and $a_{y}$ are measured variables. Since the sensor measurements of current generation of vehicle stability controller usually include $\delta, v_{x}, r$, and $a_{y}$ but not $a_{x}$, we further adapt this method to accommodate current sensor platform by approximating $a_{x}$ with vehicle speed information, i.e. $a_{x}=d u / d t$. While this approximation adds sensor measurement noise/error in the longitudinal acceleration signal, $a_{x}$, used in the observer, there are also other inevitable sensor noises in the real world which need to be considered such as vehicle speed, longitudinal and lateral acceleration measurements due to wheel slip, sensor drift, cross-talk induced by vehicle motions and road noise. In the following, we analyse the estimation performance of the modified F\&W approach (henceforth referred to as the kinematics approach) under these sensor noises, i.e. $v_{x}$, $a_{x}$, and $a_{y}$. Equation (8) can be rewritten in the form below:

$$
\left[\begin{array}{c}
\tilde{\tilde{v}}_{x} \\
\dot{\tilde{v}}
\end{array}\right]=\left[\begin{array}{cc}
-2 \alpha|r| & r \\
-\alpha r & 0
\end{array}\right]\left[\begin{array}{c}
\tilde{v}_{x} \\
\tilde{v}
\end{array}\right]+\left[\begin{array}{c}
a_{x n} \\
a_{y n}
\end{array}\right]+\left[\begin{array}{c}
-2 \alpha|r| \\
-\alpha r
\end{array}\right] u_{n} .
$$

If we use a Lyapunov function $V=\frac{\alpha^{2} \tilde{v}_{x}^{2}+\tilde{v}^{2}}{2}$, it can be seen that

$$
\dot{V}=-2 \alpha^{3}|r| \tilde{v}_{x}^{2}+\alpha^{2} \tilde{v}_{x} \cdot a_{x n}+\tilde{v} \cdot a_{y n}+\left[2 \alpha^{3}|r| \tilde{v}_{x}+\left(\alpha^{2}-1\right) r \tilde{v}\right] \cdot v_{x n} .
$$

Unlike the original analysis by $\mathrm{F} \& \mathrm{~W}, d V / d t$ is no longer negative-definite when sensor uncertainties exist, and the design parameter $\alpha$ influences the significance of sensor measurement noise. Furthermore, from Equation (9), we have $H_{a_{x n} \rightarrow \tilde{v}}=\frac{-\alpha^{2} r}{(s+\alpha|r|)^{2}}$, $H_{a_{y n} \rightarrow \tilde{v}}=\frac{s+2 \alpha|r|}{(s+\alpha|r|)^{2}}$ and $H_{v_{x n} \rightarrow \tilde{v}}=\frac{\left(\alpha^{2}-1\right) r s-2 \alpha r|r|}{(s+\alpha|r|)^{2}}$. Obviously, the design parameter/observer gain $\alpha$ influences the error propagation speed of noise in longitudinal acceleration, the sensitivity to lateral acceleration noise, and the sensitivity to vehicle speed noise.

From the analysis above, it can be seen that the steady state error contributed from our approximation of longitudinal acceleration signal is $-v$ since the approximation error we introduced is in the amount of $v r$ and the steady state gain is $\frac{1}{r}$. That is, the estimated lateral speed will eventually be washed out to zero if the derivative of vehicle speed is used in place of longitudinal acceleration. On the other hand, the estimation error during transient events (which are most critical for real-world vehicle stability control) is negligible if we keep the observer gain $\alpha$ small. Both can be concluded from the above analysis and will be verified in the following simulation section.

With real-world environment noise, a tradeoff decision has to be made for the observer gain $\alpha$ since increasing the observer gain increases the robustness to measurement noise of $a_{y}$ and $u$ but decreases the robustness (of estimation during transient events) to measurement noise of $a_{x}$. The tradeoff performance will be shown in the simulation and experimental results in the following sections. 


\section{Simulation results}

In this section the performance of the three methods are studied using simulation data taken from a nonlinear Jeep Cherokee model. The mathematical model is based on the TruckSim ${ }^{(m)}$ software (a product of the Mechanical Simulation Corporation [10]), and is constructed based on vehicle parameters published by the Vehicle Research and Testing Center of the NHTSA [11]. This TruckSim ${ }^{(i)}$ model had been verified against VRTC published test results [12] at two different vehicle speeds with steering and braking inputs that generate lateral acceleration as high as $0.6 \mathrm{~g}$.

\subsection{Flat road surface}

In the following, the TruckSim ${ }^{(m)}$ model described above is used to generate vehicle response, which is treated as the 'actual' vehicle response, based on which the three algorithms mentioned in Section 2 are verified. The steering input, vehicle forward speed and important lateral responses are shown in Figure 2, and the bird's-eye view of the vehicle trajectory is shown in Figure 3 (lane change plus a subsequent J-turn). It should be mentioned that the simulated results are obtained on flat high-friction road surface.

Figure 2 Simulation input and vehicle response

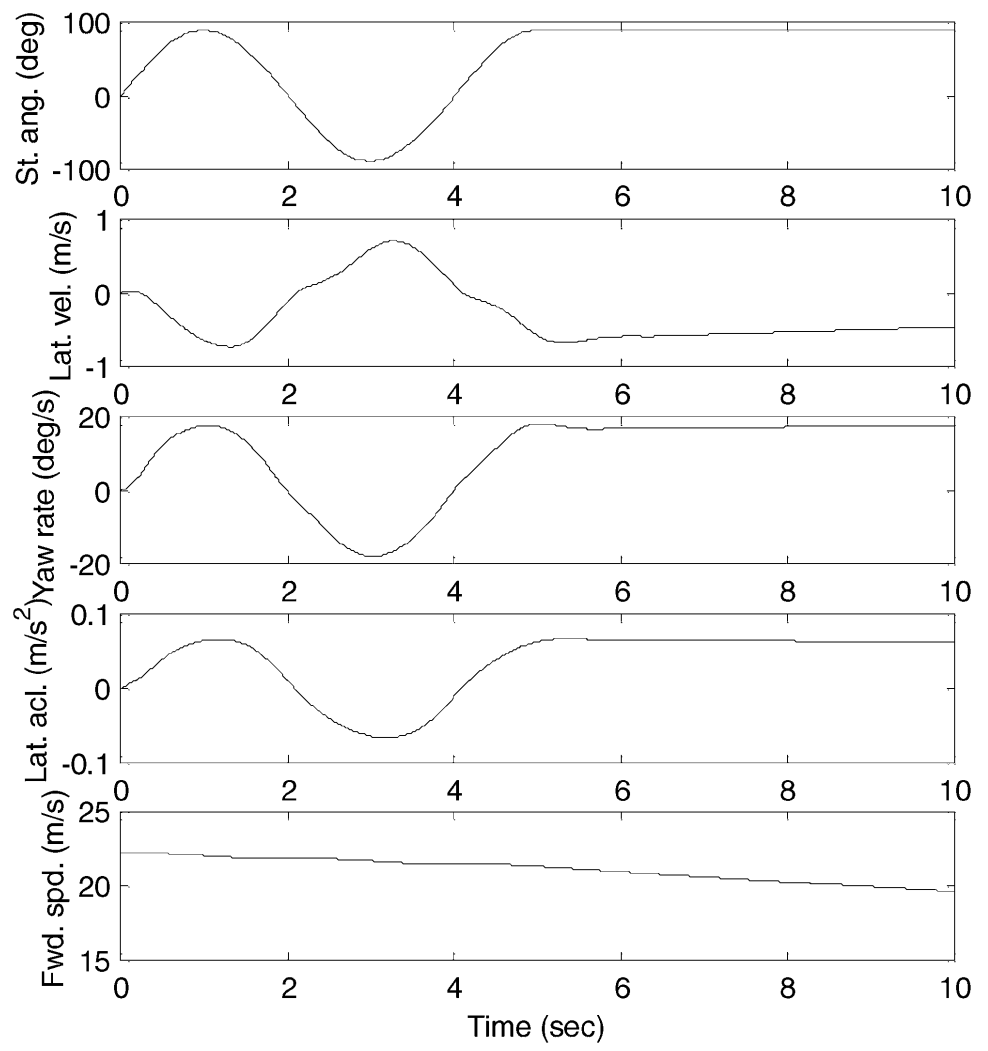


Figure 3 Bird's-eye view of the vehicle trajectory

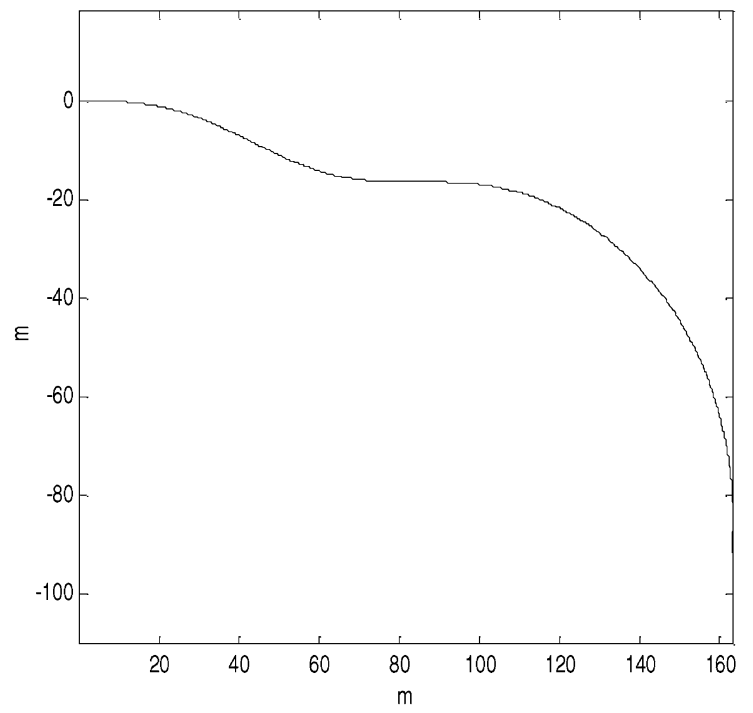

The estimation results of the transfer function method are shown in Figure 4. It can be seen that the transfer function method is very fast, and could reproduce the vehicle yaw rate response very accurately almost immediately (within a few sampling steps, i.e. $50-80 \mathrm{msec}$ ). This high accuracy, however, was not necessarily translated into accurate lateral vehicle speed estimation. If there is any inaccuracy in vehicle parameters (e.g. $m, I_{z}, a$ and $b$ ), then the tyre cornering stiffness calculation will be erroneous, which then results in inaccurate lateral speed estimation. Furthermore, since this method is model-based and does not have any adaptive capability, there is no way to compensate for any model error, other than the variations in tyre cornering stiffness. The results in Figure 4 show that the lateral speed is consistently under-estimated through most of the simulation. This may be due to the small mismatch between the vehicle parameter values used in the bicycle model and those of the TruckSim ${ }^{\circledR i}$ model. In fact, since the TruckSim ${ }^{\circledR i}$ model contains more degrees of freedom and is nonlinear, some mismatch is unavoidable.

Figure 4 Estimation results of the transfer function method (simulation)
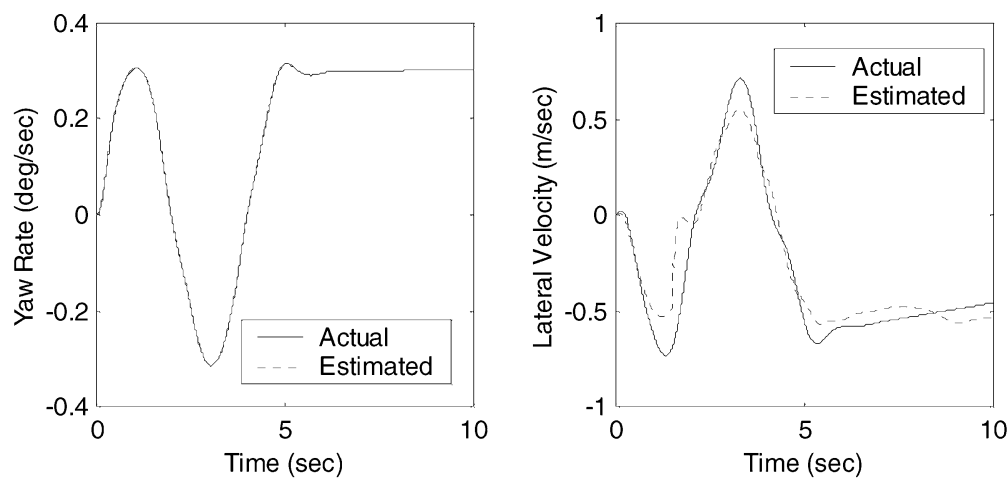
The simulation results of the state-space method are shown in Figure 5. The yaw rate estimation results are not as good as the transfer-function method, but since the yaw rate is measured anyway, that is not a problem. Since the state-space method was designed to adapt to parameter variations as well as to estimate state variables simultaneously, its estimations converge to true values slowly. In the first 1-2 seconds, the lateral speed estimation error is large. However, after the vehicle parameters are successfully adapted, the estimation becomes quite accurate. The fact it takes about 2 seconds (which depends on the observer gains $\mathrm{k}$ ) to come close to the true parameters can be seen from Figure 6. The model parameters were deliberately initialised at values away from their converged values to investigate the transient behaviour. It is quite obvious that the adaptive algorithm works but has a non-negligible convergence time.

Figure 5 Estimation results of the state-space method (simulation)
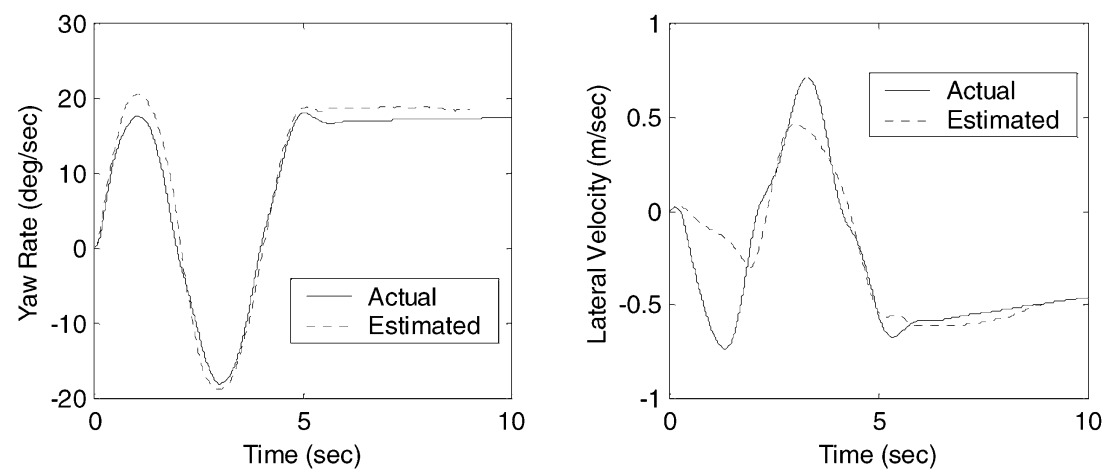

Figure 6 Estimated parameters of the state-space method (simulation)
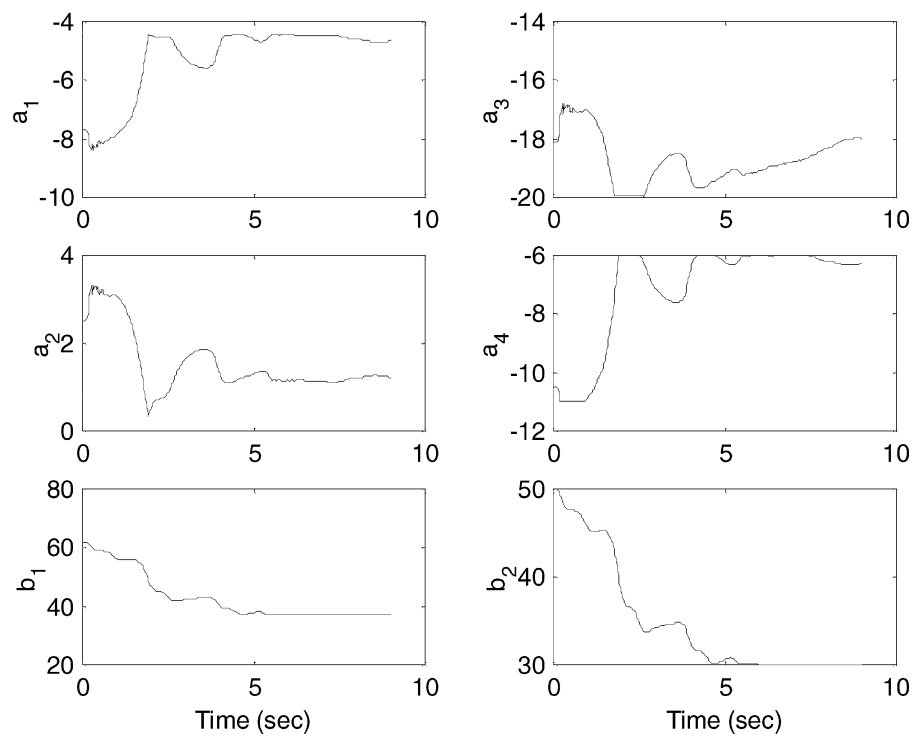
As explained in Section 2.3, the kinematics method proposed by Farrelly and Wellstead [5] is different from the other two methods. It is based on the kinematics relationship between measured signals, rather than dynamics of the vehicle. The other two methods are based on identifying input-output of vehicle handling dynamics and thus the most critical measurements are steering angle (input), road bank angle (disturbance input that needs to be estimated), vehicle forward speed (important time-varying parameter) and yaw rate (output). The F\&W method requires an additional longitudinal acceleration signal that may be subject to contamination from road gradient. We therefore adapt this method to reduce the additional sensor requirement that may not be available in a current production Active Yaw Control vehicle and approximate the longitudinal acceleration signal with vehicle speed difference, i.e. $a_{x}:=d u / d t$.

In Figure 7, multiple simulations were performed to investigate the effect of measurement noise and observer gain and to verify the analysis in Section 2.3. It can be seen from Figure 7(a) that the F\&W method has excellent performance if the sensor measurement is perfect. However, as the longitudinal acceleration is replaced by the derivative of longitudinal speed, the estimate under vehicle steady state condition will drift away from the actual while the estimate during transient manoeuvre is still adequate. It can be seen from Figure 7(a) that the larger the observer gain is applied, the sooner the steady state estimation error is realized. It is also shown that steady state estimate is indeed 'washed out' to zero with the use of longitudinal speed derivative as predicted in Section 2.3.

Figure 7 Estimation results of the kinematics method (simulation)
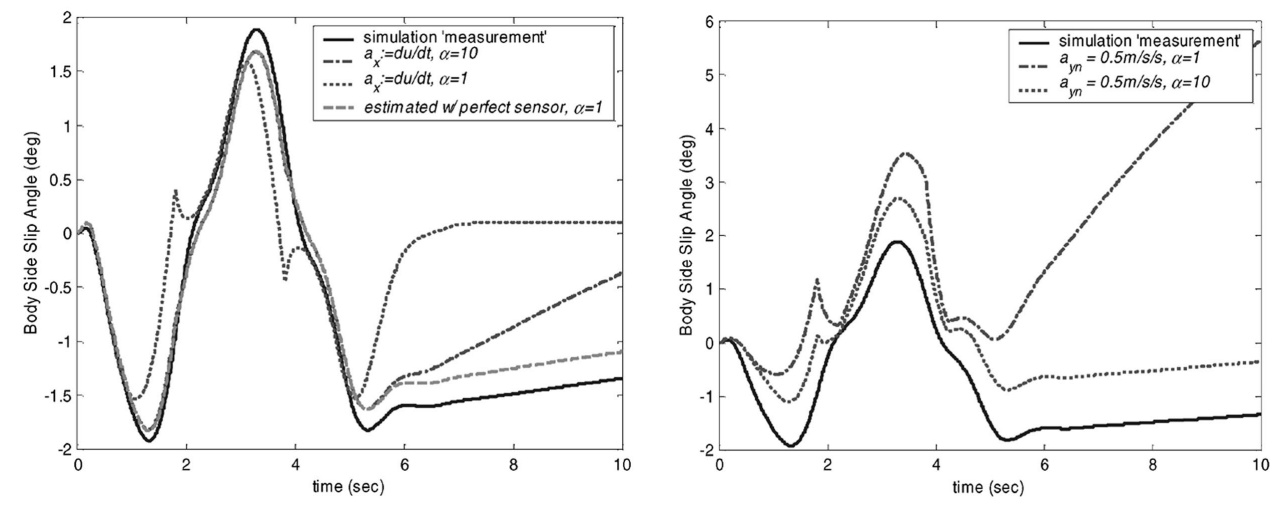

On the other hand, as the observer gain decreases, the estimate becomes more sensitive towards noise/bias in lateral acceleration measurement. The scenario where lateral acceleration contains a $0.5 \mathrm{~m} / \mathrm{s} / \mathrm{s}$ measurement bias (e.g. due to road bank angle or vehicle roll motion) is simulated and illustrated in Figure 7(b) where the effect of various observer gains is also shown. It is obvious that a trade off decision has to be made for the observer gain in order to obtain a balance between the robustness of longitudinal acceleration noise and that of lateral one. We picked an observer gain of 1 and the resulting performance will be illustrated in the experimental section where realistic and mixed sensor measurement noise is present. 


\subsection{Banked road surface}

One important implementation issue for these estimation algorithms is the robustness under real disturbances. From the algorithm formulation presented in Section 2, it can be seen clearly that all three algorithms assume zero road bank angle in the equations they used. It is not difficult to modify these basic equations to accommodate road bank angle. The real challenge, however, is a fast, robust and accurate algorithm to estimate road bank angle under a wide variety of conditions. Fortunately, this problem has been solved in a previous paper [13]. In that formulation, a three-pronged approach was proposed to estimate road bank angle. Depending on the situation, the road bank angle estimation was obtained by using vehicle input and response measurements including forward speed, steering angle, yaw rate, and lateral acceleration, that is,

$$
\hat{\phi}=f\left(u, r, a_{y}, \delta\right)
$$

Since the proposed estimation methodology is independent of lateral speed, the road bank estimate can be incorporated directly into all three models. For the transfer function method and the state-space method, the road bank angle is assumed to be an additional input to the vehicle dynamics. For the kinematics method, the road bank angle is used to remove the gravity component in lateral acceleration measurement. That is,

$$
a_{y, \text { corrected }}=a_{y}-g \sin (\hat{\phi}) .
$$

The road-bank correction was found to be necessary for all three algorithms to produce acceptable results on banked roads. In the experimental results to be presented in the next section, the road bank angle will be estimated according to the algorithm presented in [13]. Since that algorithm has been presented in detail in that paper, we will not present the equations again here.

\section{Experimental results}

In this section, the performance of the three methods are verified using test data measured from a Lincoln Mark 8 and a Mercedes S500, obtained at the Smithers Winter Test Center (low friction) and Ford Michigan Proving Ground (high friction). These tests were not designed to be repeatable under precisely controlled input or executed at fixed vehicle forward speed. Rather, the steering inputs as well as the vehicle speed were controlled by the human driver to cover a vaguely defined test matrix. Overall, there are about 40 manoeuvres executed and we selected four representative runs essentially in an arbitrary fashion, except under the case when the quality of the test data is clearly questionable. The conditions for these four tests are summarised in Table 1. It can be seen that these four runs cover nominal (flat road, high friction) as well as important adverse conditions (low friction, banked road). The vehicle side slip angle and longitudinal speed profiles are shown in Figures 8-11 for reference. The reason we chose to show side slip angle instead of steering angle is because side slip angle shows the severity of the test scenarios more clearly than the steering angle. 
Table 1 Conditions for the three tests

\begin{tabular}{llllll}
\hline & Speed & Friction & Steering & Side slip angle & Road \\
\hline Case 1 & {$[18,21] \mathrm{m} / \mathrm{sec}$} & High & Slalom & {$[-6,8] \mathrm{deg}$} & Flat \\
Case 2 & {$[15,20] \mathrm{m} / \mathrm{sec}$} & High & Slalom & {$[-3.5,4] \mathrm{deg}$} & Banked \\
Case 3 & {$[13,17] \mathrm{m} / \mathrm{sec}$} & Low & Fish-hook & {$[-5,18] \mathrm{deg}$} & Flat \\
Case 4 & {$[11,18] \mathrm{m} / \mathrm{sec}$} & Low & Sinusoidal & {$[-7,8] \mathrm{deg}$} & Flat \\
\hline
\end{tabular}

Figure 8 Speed and side slip angle of Case 1
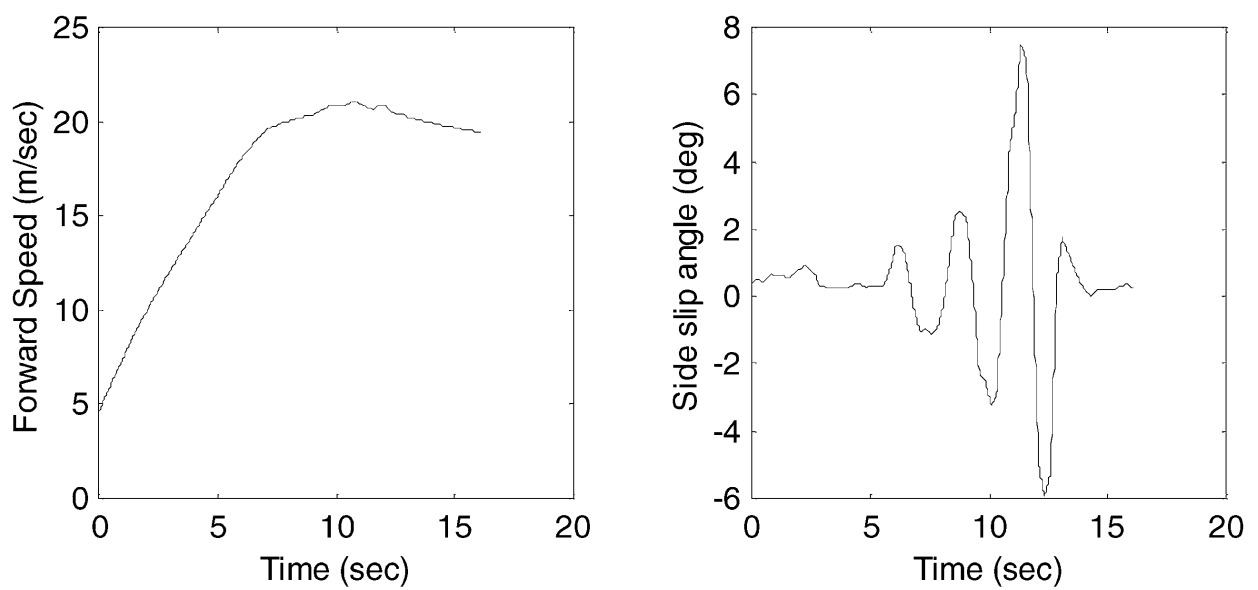

Figure 9 Speed and side slip angle of Case 2
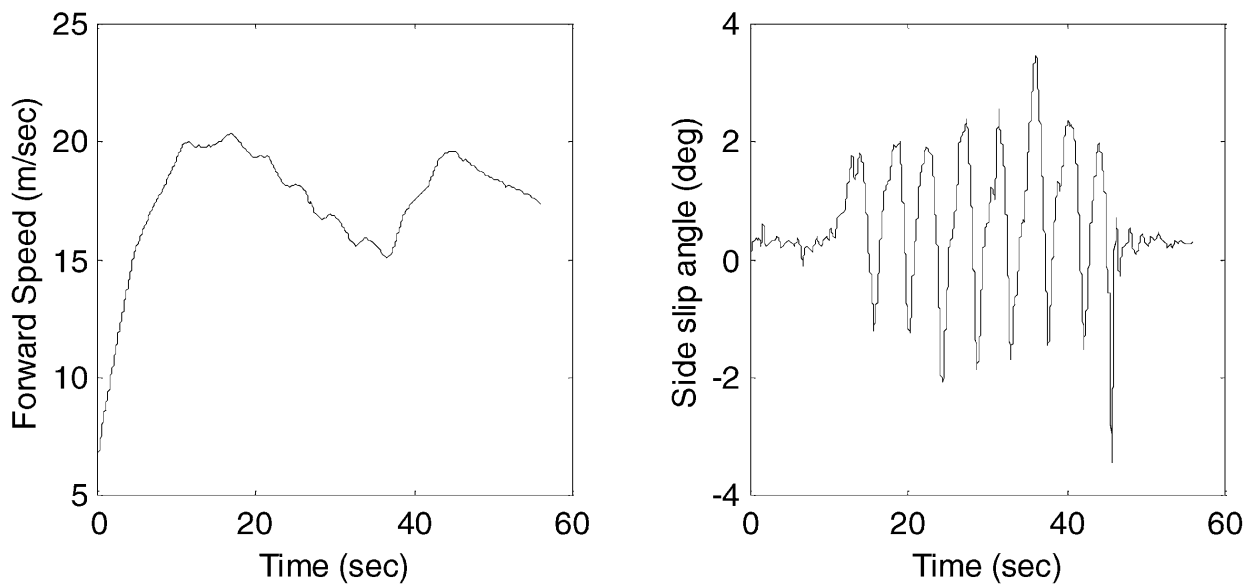
Figure 10 Speed and side slip angle of Case 3
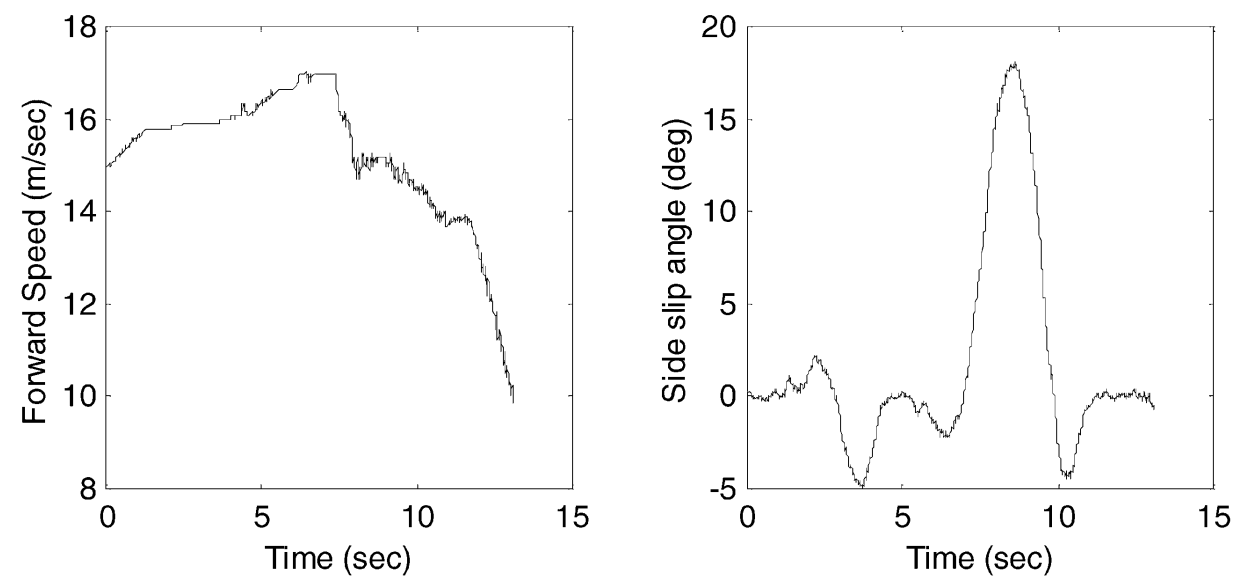

Figure 11 Speed and side slip angle of Case 4
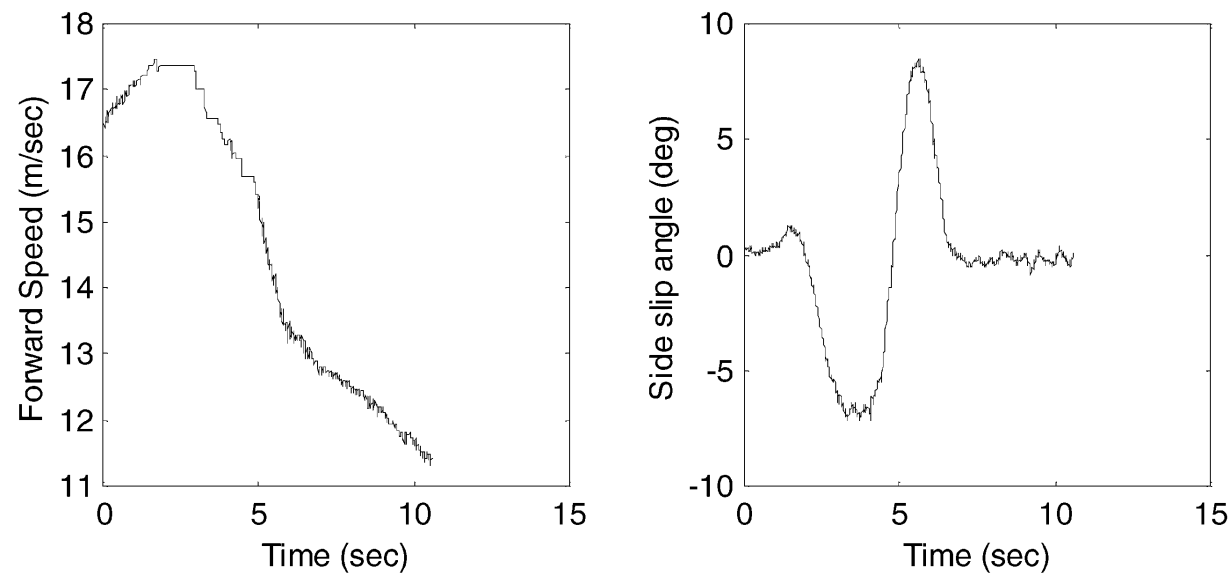

Estimation results of the three methods for the Case 1 (flat road, high friction) data are shown in Figure 12. It can be seen that the transfer function method performs worst, and the kinematics method and the state-space method are both satisfactory. This test, being performed on high-friction flat road surface, is perhaps a very easy test. There are no significant uncertainties such as road bank angle and low tyre cornering stiffness. The transfer function method consistently over-estimated the lateral speed, perhaps due to the fact that in this manoeuvre, steering input is producing very high lateral acceleration on the high friction surface. Due to the linear nature of the transfer function method, it perceives a higher lateral speed generation than that which is really generated. The performance of the kinematics method is generally good. During the second half cycle, however, it produces very large transient error due to the erroneous phase prediction. The state-space method has a very noticeable lag in the beginning but performs satisfactorily otherwise. 
Figure 12 Experimental results (Case 1)

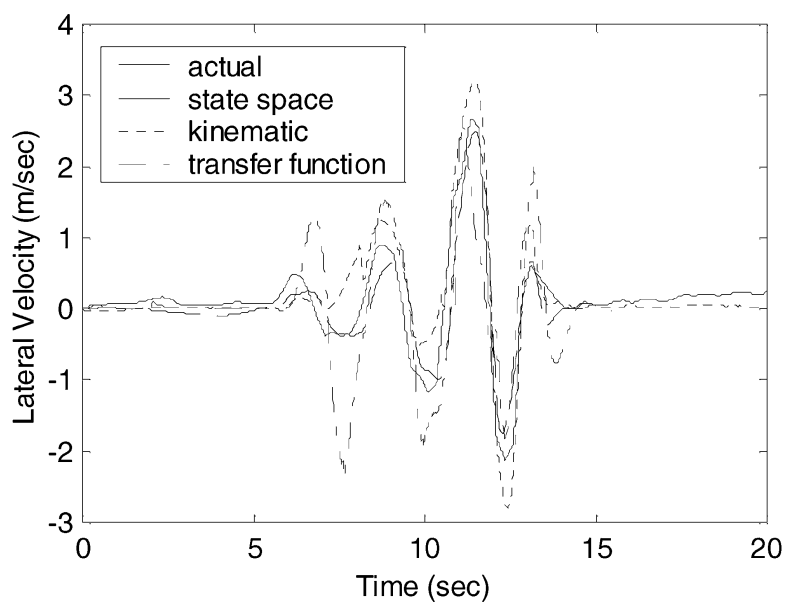

The results for Case 2 (high friction, banked road) are shown in Figure 13. In this case, the kinematics method performs worst. This is perhaps due to the fact that the estimated bank angle is only used to remove its effect on lateral acceleration measurement. It is possible that this treatment (sensor bias) is not as accurate as that which was used in the other two methods (treated as extra input). This biased estimation caused an average error of about $1 \mathrm{~m} / \mathrm{sec}$, which is quite high. The second peak in the estimated signal (at about $\mathrm{t}=20$ second) is due to a corresponding peak of the acceleration measurement. This also shows another possible issue of this method - vulnerable to noise in the acceleration measurement. The state-space method works well in the beginning but has an apparent under-estimation starting from about $\mathrm{t}=37$ second. This is perhaps due to the sudden acceleration in the longitudinal direction. Overall, the transfer function method performs the best.

Figure 13 Experimental results (Case 2)

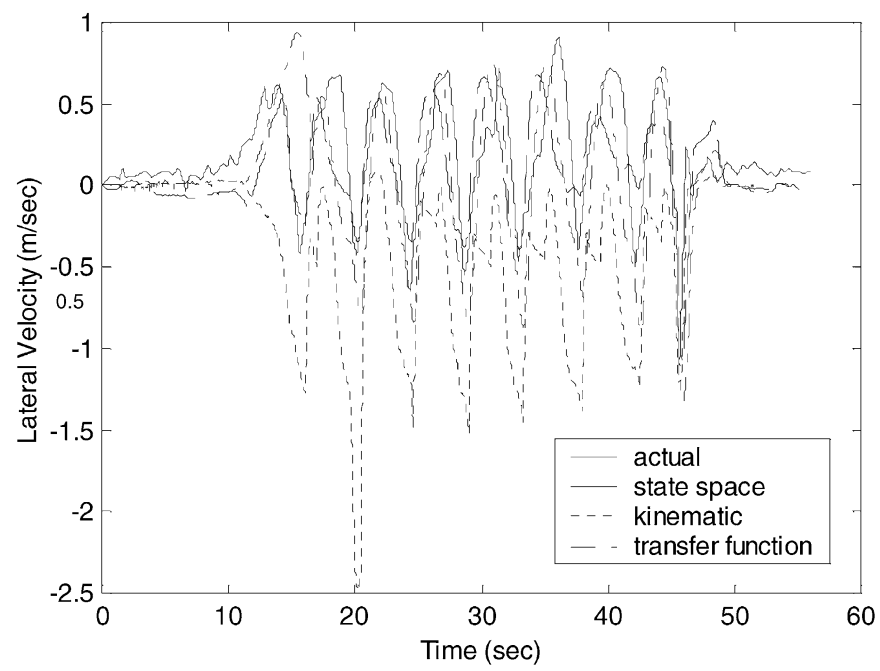


Case 3 and Case 4 (flat, icy road) are perhaps significantly more demanding than the first two cases. In these cases, the vehicle behaviour might deviate significantly from the underlying bicycle model assumption. Since the peak vehicle slip angle is close to 20 degrees in Case 3, the small angle assumption breaks down and the 'tyre cornering stiffness' can change significantly due to the simultaneous lateral and longitudinal accelerations. These deviations from underlying linear assumption not only influence the state-space and transfer function methods which rely on linear models, but also the kinematics method due to the neglected nonlinear dynamic terms. All three methods are qualitatively close to the actual vehicle response (see Figure 14) but produce large transient errors for the last four peaks (at $t=5,6.5,8.5$ and $10 \mathrm{sec}$ ). All three methods could over-predict $(t=5,6.5,10 \mathrm{sec})$ or sometimes under-predict $(\mathrm{t}=8.5 \mathrm{sec})$. This inconsistent behaviour might be due to vehicle roll motion and resulted measurement error. It is possible a more complicated model (yaw-roll) is necessary to represent the vehicle dynamics more accurately. This issue is currently under investigation. Overall, the kinematics method seems to work better, but its transient error can still be as large as $2.5 \mathrm{~m} / \mathrm{sec}$. It is fair to say that all three algorithms fail to achieve a satisfactory score for this Case.

Figure 14 Experimental results (Case 3)

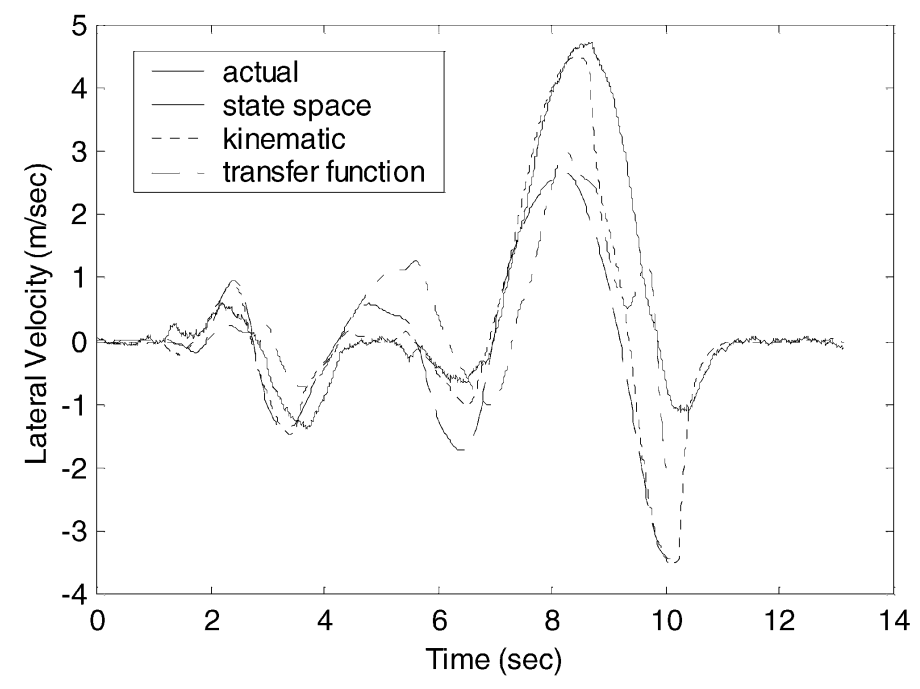

Even though the peak side slip angle of the Case 4 results is not as high as that of Case 3, it is subjectively perceived as a more 'out-of-control' scenario by the driver due to its fast reversing steering action. The overall test lasts for less than 6 seconds, $40 \%$ shorter than the Case 3 scenario. Again, the kinematics method performs the best, due to the fact its response is fast. The transfer function method demonstrates very unacceptable performance, which is surprising given the fact it performs rather reasonably in Case 3. In fact we observe several other 'unexpected failures' of the transfer function method when we examine the results from other test cases. This inconsistent behaviour is a concern about this approach. The state-space method, due to the nature of its slower response compared with the other two methods, fails 
to catch the first (down) peak, and the estimation for the second (up) peak is noticeably slower than the actual signal. The slow response is definitely a major problem of this algorithm.

Figure 15 Experimental results (Case 4)

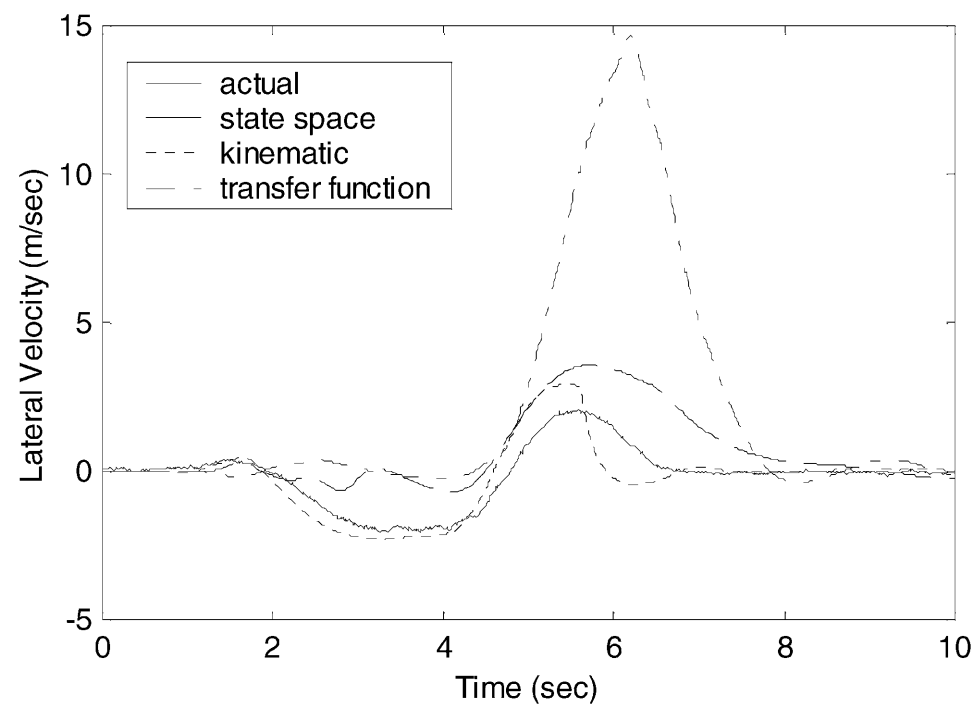

\section{Conclusions}

Three methods were used to estimate vehicle lateral speed during transient manoeuvres. We found that it is necessary to have a separate algorithm to provide an accurate road bank angle estimation which, if not compensated for, will produce unacceptable results for all three algorithms on banked roads. We have also tried artificial if-then rules to tweak performance under road bank, however, performance under other scenarios will be sacrificed and these 'patches' may not be moved to other designs easily. Therefore, we implemented the road-bank estimation algorithm previously developed by Tseng [13]. All three methods were shown to work well on high friction, flat road surfaces. On various non-nominal road surfaces, all three methods are able to predict the trend of the lateral speed but there are still some significant differences between the estimate and the actual vehicle response at times. We suspect a further blending of three approaches might be necessary to produce better and more robust estimation at all time. Overall, the kinematics algorithm seems to be most robust, except on banked road surfaces. The major problem of the state-space method is its slow response due to its simultaneous estimation of parameters and state variables. The transfer function performs surprisingly well for many cases, but could produce very poor estimation unexpectedly. It is fair to say that none of these algorithms is a clear winner through this comparison study. Enhancement to improve their respective weakness is necessary before they can be used alone, or as an integrated system to produce reliable lateral speed estimation. 


\section{Acknowledgement}

The authors would like to thank Dr Davor Hrovat at Ford Research Laboratory for his invaluable insight, suggestions, and support for this study. We would also like to acknowledge John Yester and Mitch McConnell at Ford Research Laboratory for their help in performing the vehicle experiments.

\section{References and Notes}

1 Senger and Kortum (1989) 'Investigations on state observers for the lateral dynamics of four wheel steered vehicles', The Dynamics of Vehicles on Roads and Tracks, Supplement to Vehicle System Dynamics, Vol. 18, pp.515-527.

2 Cao (1994) 'Method of obtaining the yawing velocity and/or transverse velocity of a vehicle', US Patent No. 5311431, May.

3 Kaminaga, M. and Naito, G. (1998) 'Vehicle body slip angle estimation using an adaptive observer', Proceedings of the 4th International Symposium on Advanced Vehicle Control ( $A V E C)$, Nagoya, Japan, 14-18 September, pp.207-212.

4 Liu, C. and Peng, H. (1998) 'A state and parameter identification scheme for linearly parameterized systems', ASME Journal of Dynamic Systems, Measurement and Control, Vol. 120, No. 4, pp.524-528.

5 Farrelly, J. and Wellstead, P. (1996) 'Estimation of vehicle lateral velocity', Proceeding of the 1996 IEEE International Conference on Control Applications, Dearborn, MI, 15-18 September, pp.552-557.

6 Fukada, Y. (1998) 'Estimation of vehicle side-slip with combination method of model observer and direct integration', Proceedings of the 4th International Symposium on Advanced Vehicle Control (AVEC), Nagoya, Japan, 14-18 September, pp.201-206.

7 Nishio A., et al. (2001) 'Development of vehicle stability control system based on vehicle sideslip angle estimation', 2001 SAE World Congress, SAE Paper 2001-01-0137.

8 Sasaki, H. and Nishimaki, T. (1989) 'A side-slip angle estimation using neural network for a wheeled vehicle', 2000 SAE World Congress, SAE Paper 2000-01-0695.

9 Hac, A. and Simpson, M.D. (2000) 'Estimation of vehicle side slip angle and yaw rate', 2000 SAE World Congress, SAE Paper 2000-01-0696.

10 http://www.trucksim.com/, Ann Arbor, MI 48103, USA.

11 Salaani, M.K., Guenther, D.A. and Heydinger, G.J. (1999) 'Vehicle dynamics modeling for the national advanced driving simulator of a 1997 jeep cherokee', SAE Paper No. 1999-01-0121.

12 Chen, B. and Peng, H. 'A rollover warning algorithm for sports utility vehicles', Proceedings of the 1999 American Control Conference, San Diego, CA.

13 Tseng, H.E. (2000) 'Dynamic estimation of road bank angle', Proceedings of the 5th International Symposium on Advanced Vehicle Control (AVEC), Ann Arbor, MI, USA, 22-24 August, pp.421-428. 